Relations industrielles

Industrial Relations

\title{
Recrutement et sélection du personnel Par Anne Bourhis. Montréal : G. Morin, 2007, 562 p., ISBN 978-2-891059-89-3.
}

\section{François Bernard Malo}

Volume 64, numéro 1, hiver 2009

URI : https://id.erudit.org/iderudit/029550ar

DOI : https://doi.org/10.7202/029550ar

Aller au sommaire du numéro

Éditeur(s)

Département des relations industrielles de l'Université Laval

ISSN

0034-379X (imprimé)

1703-8138 (numérique)

Découvrir la revue

Citer ce compte rendu

Malo, F. B. (2009). Compte rendu de [Recrutement et sélection du personnel Par Anne Bourhis. Montréal : G. Morin, 2007, 562 p., ISBN 978-2-891059-89-3.]

Relations industrielles / Industrial Relations, 64(1), 177-179.

https://doi.org/10.7202/029550ar

Tous droits réservés @ C Département des relations industrielles de l'Université Laval, 2009
Ce document est protégé par la loi sur le droit d'auteur. L’utilisation des services d'Érudit (y compris la reproduction) est assujettie à sa politique d'utilisation que vous pouvez consulter en ligne.

https://apropos.erudit.org/fr/usagers/politique-dutilisation/ 
discusses how the misplaced concern as to the ability of the CPP to cope with the retirement of the baby boomers was played upon by those with a commercial interest in selling allied financial products, but was allayed by the Chief Actuary demonstrating that contribution increases taken earlier for this purpose meant that, even at current contribution levels, the fund would be viable for at least 75 years.

These initial pension-related concerns gave way to a more general economic concern; the need to keep the boomers working longer to ensure the economy's capacity to maintain, or enhance, the requisite standard of living for all. The author describes how thought, influenced by neo-liberal forces, has moved away from the former welfare state model. Instead, "life-course flexibility" and "active aging" have become the new buzz words for policy-makers. Proponents of these approaches, including the OECD, contend more "choice" and "flexibility" would be afforded workers in relation to how, and when, they choose to allocate their life-span time between working, learning, caring and leisure. Townson contends that "choice" and "flexibility" are, in fact, code words for expecting workers to fend for themselves much more than they do now, requiring them-rather than their employers - to assume the market risks associated with their retirement savings investments. Introduced first by Prime Minister Tony Blair in Britain, the "Third Way", also described as the post-welfare state, reinforced the trend, with its emphasis on the individual's responsibility to provide for retirement rather than society as whole.

With debate about the requirement to increase the retirement age and remove mandatory retirement a need to protect older workers is advanced. This involves a wideranging review of the attitudinal, social and economic factors determining the timing of workers "retirement", in which the need to recognize the thread that links all of the relevant government policy areas is delineated. Discrimination against the elderly is well covered, pointing to the need for appropriate government policy development. Such policy needs to meet two objectives: to promote workplace practices which are conducive to the ongoing employment of the elderly; and to alleviate perceptions of coercion among the elderly in the new "active aging" approach.

The special needs of women are also well handled. Townson discusses their increasing participation in the paid work force and, owing to their primary care-giver role both to children and the elderly, their tendency to "retire" earlier, and to take breaks from work or pass up promotional opportunities more frequently than men do. These factors, she contends, must be considered when proposing amendments to retirement policy.

Due to her mastery of the subject matter and her skill as a writer Townson has managed to present a book on such a complex subject, exploring the many diverse factors and the sometimes conflicting needs of individuals, governments, employers and unions, in a manner which is comprehensible to the general reader.

The only minor complaints I have relate to editing of the book. I found several typographical errors, and tables or charts were sometimes too far removed from the initial reference to them. Also, as a non-Canadian reader, I found the use of acronyms before first using the name in full to be somewhat distracting - particularly in the first chapter. In view of this I consider that the book would benefit from the addition of a list of acronyms and their related full titles.

Richard R. Gordon

Brisbane, Australia

\section{Recrutement et sélection du personnel}

Par Anne Bourhis. Montréal : G. Morin, 2007, 562 p., ISBN 978-2-891059-89-3.

Cet ouvrage d'Anne Bourhis vient combler un grand vide dans le domaine de la publication, en langue française, d'ouvrages se concentrant sur la dotation. Jusqu'à tout récemment, le lecteur intéressé par ces questions devait se contenter d'ouvrages périphériques ou encore, d'ouvrages marqués par le passage des années ou les travers d'une approche de consultants.

Divisé en cinq parties comprenant au total dix-huit chapitres, la contribution de Bourhis est intéressante à plusieurs niveaux. Tout 
d'abord, remarquons que la plupart des chapitres comportent des statistiques pertinentes ou des exemples actuels permettant d'avoir une bonne idée de l'ensemble de la question en contexte québécois. Ensuite, chacun des chapitres est structuré à l'aide de grands thèmes qui illustrent la diversité des notions abordées de même que les perspectives qu'il est permis d'emprunter pour mieux appréhender le sujet. Enfin, la grande majorité des chapitres comportent de nombreux liens internet ainsi qu'une bibliographie (cependant trop sommaire et regroupant peu de publications scientifiques récentes) qui permettent aux lecteurs intéressés à en savoir davantage de se lancer sur quelques pistes déjà bien balisées.

La première partie du livre s'intitule « Les enjeux de la dotation » et elle regroupe trois chapitres (Les enjeux du marché du travail; L'encadrement juridique des activités de dotation et Se positionner comme employeur de choix). Alors que le premier chapitre séduit par le vaste panorama présenté et le troisième, par l'immense actualité du sujet abordé, le deuxième chapitre sur l'encadrement juridique des activités de dotation est quant à lui illustré de cas pertinents de jurisprudence et présente d'une manière agréable la situation du Québec par rapport à celle des autres provinces canadiennes et à la législation fédérale.

La deuxième partie de l'ouvrage comporte pour sa part quatre chapitres (La planification des ressources humaines; L'analyse de fonction et la description de poste; Le profil de compétences et les critères de sélection et La planification des activités de dotation). De façon générale ces divers chapitres sont tous très instructifs et très bien écrits. Nous avons particulièrement apprécié l'effort de l'auteure pour présenter (dans son sixième chapitre) divers outils de pondération et de classification des compétences de même qu'un exemple concret permettant aux étudiants de réaliser le caractère très pratique de la démarche. Ceci dit, nous aurions apprécié que l'auteure fasse une plus grande place à la planification stratégique des ressources humaines (dans son troisième chapitre) de même qu'aux distinctions théoriques de plus en plus importantes entre la logique de poste et la logique de compétence (tissant ainsi un maillage plus serré entre les chapitres 5 et 6).
La troisième partie du livre s'intitule quant à elle « Le recrutement » et elle comporte trois chapitres (Les méthodes de recrutement; Le recrutement international et La rédaction d'une annonce). Encore une fois, l'ouvrage témoigne d'un souci constant de vulgariser le sujet, de le rendre opérationnel pour un étudiant de premier cycle en relations industrielles et en gestion des ressources humaines et d'en saisir le panorama le plus large possible.

La quatrième partie de l'ouvrage, intitulée "La sélection », comporte cinq chapitres (Les fondements de la mesure; La présélection; L'entrevue de sélection; Les tests de sélection et La vérification des antécédents). De toutes les parties de l'ouvrage, c'est sans conteste la plus importante et celle sur laquelle de nombreux autres auteurs ont publié au cours des dernières années. En dépit d'une concurrence beaucoup plus forte que celle présente pour les autres parties du livre, cette quatrième partie s'en tire encore une fois fort bien. Les chapitres 13 et 14 (sur l'entrevue et les tests de sélection) sont particulièrement bien structurés et très bien vulgarisés. Bien entendu certains sujets auraient pu être traités plus amplement (pensons notamment aux caractéristiques scientifiques des divers instruments de mesure et à l'acte d'évaluer le potentiel humain), mais il ne faut pas perdre de vue qu'il s'agit ici d'une publication sur l'ensemble de la dotation du personnel et non seulement sur la sélection de celui-ci.

La cinquième et dernière partie du livre s'intitule " L'embauche et l'intégration en emploi » et elle comporte trois chapitres (L'embauche; L'accueil et l'intégration des nouveaux employés et L'évaluation du processus de dotation). Comme pour tous les autres chapitres de I'ouvrage, ces derniers sont bien écrits, abondamment illustrés et ils témoignent tous d'un souci de rendre compréhensible pour le futur praticien en quoi consiste le travail d'un spécialiste de la dotation du personnel. Bien entendu il aurait pu être souhaitable de discuter plus à fond de la théorie de la prise de décision lorsque vient le temps de préparer l'offre d'embauche mais encore une fois, il ne faut pas perdre de vue qu'il s'agit ici d'un ouvrage général et non d'un manuel spécialisé sur cette question particulière. Dans cette dernière partie nous avons particulièrement aimé le dix-huitième chapitre portant sur l'évaluation du processus de dota- 
tion. II permet, à notre avis, de faire réaliser à l'étudiant qu'en l'absence de suivi, toute prise de décision est nécessairement incomplète, voire complètement hasardeuse.

De façon générale, il est donc possible de dire que la contribution d'Anne Bourhis est de très bonne qualité et qu'elle mérite sans l'ombre d'un doute de faire partie de la bibliothèque de tout futur gestionnaire en ressources humaines.

Si nous avions quelques commentaires généraux à émettre à l'auteure pour bonifier une prochaine édition, nous les regrouperions sous une seule rubrique : la distanciation critique. Dans une prochaine édition, nous aimerions que l'auteure questionne de façon un peu plus serrée certaines des réalités avancées et des solutions préconisées par les gestionnaires pour y faire face. À titre d'exemple, l'auteure écrit dans le premier chapitre de son ouvrage que depuis quelques années nous assistons à une augmentation notable du taux d'activité. Nous aurions aimé qu'elle prenne le soin d'expliquer les motifs économiques et les choix politiques qui ont servi à modifier la réalité des personnes auparavant sans emploi officiel. Si l'expert sait que la chose s'explique en partie par la réduction drastique des protections sociales de la part des différents paliers de gouvernement inspirés par une idéologie remettant fondamentalement en cause l'État-providence (par le biais de la réduction de l'accessibilité à l'assurance emploi et aux prestations de soutien du revenu) et ses mesures passives de soutien au développement économique, il est plus qu'improbable que le néophyte en ait déjà été mis au parfum.

Toujours dans la même veine l'auteure présente, dans son troisième chapitre, l'importance qu'a récemment prise la nécessité pour les organisations de se faire reconnaître comme des employeurs de choix parmi les chercheurs d'emploi. Si le phénomène est bien réel, nous aurions apprécié que Bourhis n'hésite pas à s'attaquer à la multiplicité des concours de ce type et aux causes qui sous-tendent le phénomène. Comment peut-on expliquer, par exemple, que des organisations comme Wal-Mart et McDonald's qui sont généralement réputées auprès de la communauté des spécialistes en gestion des ressources humaines comme des employeurs plutôt lambda finissent régulièrement en tête de ces divers palmarès ? Sommes-nous, en tant qu'experts en ressources humaines, en face d'une réalité que l'on ignore ou d'une image trafiquée par quelques ramassis de capitalistes mal intentionnés ?

\section{François Bernard Malo}

Université Laval

\section{Social Marketing: Influencing Behaviors for Good}

By Philip Kotler and Nancy R. Lee. Los Angeles: Sage Publications, 2008, 446 pp. +1 CD, $3^{\text {rd }}$ ed. ISBN 978-1-412956-47-5 (pbk.: alk. paper).

This book, by Philip Kotler and Nancy R. Lee, is a blueprint for organizations with an interest in developing and implementing programs for influencing public behaviours. It is very useful for those engaged in community and not for profit activities and, indeed, many of the case studies contained in the book focus on the impact that such programs have had in modifying social behaviour.

Kotler and Lee's contention is that it is considerably more difficult to sell behaviour modification than goods or services. For example, how does one convince people to spend more money or engage in uncomfortable or unfamiliar activities? This book provides many examples of how it is possible and it sets out the basics of how it may be achieved, down to obtaining funding for awareness campaigns.

The majority of the case studies centre around health and environment and this publication demonstrates that social marketing can have a significant impact upon a wide variety of issues. The book demonstrates the critical importance of transforming attitudes to matters of concern such as suicide (we are informed that $9.4 \%$ of $9^{\text {th }}-12^{\text {th }}$ graders attempted suicide one or more times during the past 12 months), binge drinking, school and domestic violence, HIV/AIDs and animal adoption (10 million unwanted animals in animal shelters are euthanized each year).

Examples vary from those in their infancy such as the impact of the prevention of methamphetamine use project in Montana to those which have had a measurable and positive impact on communities. Research shows encouraging results in campaigns designed persuade the public to wear seatbelts, refrain from littering, use water 